\title{
Triple sums including Fibonacci numbers with three binomial coefficients
}

\author{
Funda Taşdemir \\ Department of Mathematics, Yozgat Bozok University \\ Yozgat, Turkey \\ e-mail: funda.tasdemireyobu.edu.tr
}

\begin{abstract}
In this paper, we consider some triple sums that involve Fibonacci numbers with three binomial coefficients. We chose the indices of Fibonacci numbers as linear combination of the summation indices. Moreover, various types of alternating analogues of them whose powers depend on the index or indices are computed. These sums are evaluated in nice multiplication forms in terms of Fibonacci and Lucas numbers.
\end{abstract}

Keywords: Fibonacci numbers, Lucas numbers, Binomial triple sums.

2010 Mathematics Subject Classification: 11B39, 05A10.

\section{Introduction}

The generalized second order sequences $\left\{U_{n}\right\}$ and $\left\{V_{n}\right\}$, are defined for $n>0$ and nonzero integer number $p$ by $U_{n+1}=p U_{n}+U_{n-1}$ and $V_{n+1}=p V_{n}+V_{n-1}$, in which $U_{0}=0, U_{1}=1$ and $V_{0}=2, V_{1}=p$, respectively. If $p=1$, then $U_{n}=F_{n}$ ( $n$-th Fibonacci number) and $V_{n}=L_{n}$ ( $n$-th Lucas number). The Binet formulas for these sequences are given by

$$
F_{n}=\frac{\alpha^{n}-\beta^{n}}{\alpha-\beta} \text { and } L_{n}=\alpha^{n}+\beta^{n}
$$

where $\alpha$ and $\beta$ are $(1 \pm \sqrt{5}) / 2$. By the Binet formulas for $F_{n}$ and $L_{n}$,

$$
F_{-n}=(-1)^{n+1} F_{n} \text { and } L_{-n}=(-1)^{n} L_{n} .
$$


Many identities related to binomial or generalized binomial coefficients, the numbers obtained by using linear homogeneous recurrence relations have been extensively studied in great detail by many authors such as $[1-4,12,13,15-19]$. Carlitz [1] considers some sums that involve Fibonacci or Lucas numbers with one binomial coefficient. Kılıç et. al. [9] introduce the sums and alternating sums of products of terms of sequences $\left\{U_{k n}\right\}$ and $\left\{V_{k n}\right\}$ with binomial coefficients. They study the sums have the form:

$$
\sum_{i=0}^{n}\left(\begin{array}{l}
n \\
i
\end{array}\right) U_{k(a+b i)} V_{k(c+d i)} \text { and } \sum_{i=0}^{n}\left(\begin{array}{l}
n \\
i
\end{array}\right)(-1)^{i} U_{k(a+b i)} V_{k(c+d i)} .
$$

Kiliç et. al. [6] prove formulas for the sums and alternating sums of powers of terms of sequences $\left\{F_{n}\right\}$ and $\left\{L_{n}\right\}$ with binomial coefficients. Kılıç and Belbachir [8] consider various double binomial sums related to certain second-, third- and fourth-order recursions. Also they derive a new binomial sums with complex coefficients related with a generalized second order recursion. Kılıç and Arıkan [7] present many interesting sums with two binomial coefficients. As double binomial sums examples, they present the following new special results:

$$
\sum_{0 \leq i, j \leq n}\left(\begin{array}{c}
n-i \\
j
\end{array}\right)\left(\begin{array}{c}
i+j \\
j
\end{array}\right)(-1)^{i}=F_{n+1} \quad \text { and } \sum_{0 \leq i, j \leq n}\left(\begin{array}{c}
n+i \\
2 j
\end{array}\right)\left(\begin{array}{l}
i \\
j
\end{array}\right) 2^{j}=F_{3 n+1} .
$$

Kılıç and Taşdemir [10] compute various sums including $\left\{U_{n}\right\}$ or $\left\{V_{n}\right\}$ with one binomial coefficient. Kılıç and Taşdemir [11] also derive some different special binomial and alternating binomial double sums with the Fibonacci numbers. Taşdemir and Toska [21] consider some special binomial and alternating binomial double sums with the Lucas numbers. For example, for $t \neq-1$, they present the following result:

$$
\sum_{0 \leq i, j \leq n}\left(\begin{array}{l}
i \\
j
\end{array}\right) L_{(4 t+2) i+j}=\frac{L_{2 n(t+1)} F_{2(n+1)(t+1)}}{F_{2(t+1)}} .
$$

Recently, Ömür and Duran [14] compute binomial triple sums with two binomial coefficients. They consider certain binomial triple sums given by

$$
\sum_{0 \leq i, j, k \leq n}\left(\begin{array}{l}
i \\
j
\end{array}\right)\left(\begin{array}{l}
j \\
k
\end{array}\right) F_{r i+j+k}
$$

More recently, Taşdemir [20] consider various binomial and alternating binomial triple sums including two binomial coefficients and the Lucas numbers. As a binomial triple sum example, the following new result is given:

$$
\begin{aligned}
& \sum_{0 \leq i, j, k \leq n}\left(\begin{array}{l}
i \\
j
\end{array}\right)\left(\begin{array}{l}
j \\
k
\end{array}\right) L_{(4 t+1) i+j+k}=\frac{1}{3+2 L_{4 t+3}} \\
& \times \begin{cases}2^{n+1}\left(2 L_{\frac{(4 t+3) n}{2}} L_{\frac{(4 t+3) n}{2}}+5 F_{\frac{(4 t+3)(n+1)-3}{2}} F_{\frac{(4 t+3)(n+1)+3}{2}}\right)+2 L_{2 t+1} L_{2 t+2} & \text { if } n \text { is even, } \\
2^{n+1}\left(10 F_{\frac{(4 t+3) n+1}{2}} F_{\frac{(4 t+3) n-1}{2}}+L_{\frac{(4 t+3)(n+1)}{2}} L_{\frac{(4 t+3)(n+1)}{2}}\right)+2 L_{2 t+1} L_{2 t+2} & \text { if } n \text { is odd. }\end{cases}
\end{aligned}
$$

In this study, the binomial triple sums involving three binomial coefficients and the Fibonacci numbers are considered. Besides, some alternating analogues of them whose powers depend on the index or indices are computed. Moreover, these sums we shall compute are evaluated in nice multiplication form in terms of again $\left\{F_{n}\right\}$ and $\left\{L_{n}\right\}$. 


\section{Binomial triple sums with the Fibonacci numbers}

In this section, we will present some new triple binomial sums. Before them, we will need the following two auxiliary lemmas for further use.

Lemma 2.1. For any real numbers $x, y$ and $z$

$$
\sum_{0 \leq i, j, k \leq n}\left(\begin{array}{l}
n \\
i
\end{array}\right)\left(\begin{array}{l}
i \\
j
\end{array}\right)\left(\begin{array}{l}
j \\
k
\end{array}\right) x^{i} y^{j} z^{k}=(1+x+x y+x y z)^{n} .
$$

Proof. From Binomial Theorem, it can be clearly obtained.

Also we give the following lemma without proof that could be easily derived from the Binet formulas for Fibonacci and Lucas numbers (see [22] ).

Lemma 2.2. Let $t$ be an integer. Then,

$$
\begin{aligned}
1+(-1)^{t} \alpha^{2 t} & =(-1)^{t} \alpha^{t} L_{t}, \\
1+(-1)^{t} \beta^{2 t} & =(-1)^{t} \beta^{t} L_{t}, \\
1+(-1)^{t+1} \alpha^{2 t} & =(-1)^{t+1} \alpha^{t} F_{t} \sqrt{5}, \\
1+(-1)^{t+1} \beta^{2 t} & =(-1)^{t} \beta^{t} F_{t} \sqrt{5} .
\end{aligned}
$$

We can give the first of the results now.

Theorem 2.3. For nonnegative integer $n$ and integer $t$

(i) $\sum_{0 \leq i, j, k \leq n}\left(\begin{array}{l}n \\ i\end{array}\right)\left(\begin{array}{l}i \\ j\end{array}\right)\left(\begin{array}{l}j \\ k\end{array}\right) F_{i+(4 t+1) j+k}=F_{2 t+1}^{n}\left\{\begin{array}{cl}5^{\frac{n}{2}} F_{(2 t+3) n} & \text { if } n \text { is even } \\ 5^{\frac{n-1}{2}} L_{(2 t+3) n} & \text { if } n \text { is odd }\end{array}\right.$

(ii) $\sum_{0 \leq i, j, k \leq n}\left(\begin{array}{l}n \\ i\end{array}\right)\left(\begin{array}{l}i \\ j\end{array}\right)\left(\begin{array}{l}j \\ k\end{array}\right) F_{i+(4 t+3) j+k}=L_{2 t+2}^{n} F_{(2 t+4) n}$

(iii) $\sum_{0 \leq i, j, k \leq n}\left(\begin{array}{l}n \\ i\end{array}\right)\left(\begin{array}{l}i \\ j\end{array}\right)\left(\begin{array}{l}j \\ k\end{array}\right) F_{i+2 j+k}=2^{n} F_{4 n}$

(iv) $\sum_{0 \leq i, j, k \leq n}\left(\begin{array}{l}n \\ i\end{array}\right)\left(\begin{array}{l}i \\ j\end{array}\right)\left(\begin{array}{l}j \\ k\end{array}\right) F_{i+j+5 k}=5^{n} F_{4 n}$

(v) $\sum_{0 \leq i, j, k \leq n}\left(\begin{array}{l}n \\ i\end{array}\right)\left(\begin{array}{l}i \\ j\end{array}\right)\left(\begin{array}{l}j \\ k\end{array}\right) F_{i+j}=3^{n} F_{2 n}$

(vi) $\sum_{0 \leq i, j, k \leq n}\left(\begin{array}{l}n \\ i\end{array}\right)\left(\begin{array}{l}i \\ j\end{array}\right)\left(\begin{array}{l}j \\ k\end{array}\right) F_{i+k}=F_{4 n}$

Proof. We only prove the first claim. The others could be similarly proven. Consider the left-hand side of the first claim, by the Binet formula, equals

$$
\frac{1}{\alpha-\beta} \sum_{0 \leq i, j, k \leq n}\left(\begin{array}{l}
n \\
i
\end{array}\right)\left(\begin{array}{l}
i \\
j
\end{array}\right)\left(\begin{array}{l}
j \\
k
\end{array}\right)\left[\alpha^{i+(4 t+1) j+k}-\beta^{i+(4 t+1) j+k}\right],
$$


which, by Lemma 2.1, equals

$$
\begin{aligned}
& \frac{1}{\alpha-\beta}\left[\left(1+\alpha+\alpha^{4 t+2}+\alpha^{4 t+3}\right)^{n}-\left(1+\beta+\beta^{4 t+2}+\beta^{4 t+3}\right)^{n}\right] \\
= & \frac{1}{\alpha-\beta}\left[(1+\alpha)^{n}\left(1+\alpha^{4 t+2}\right)^{n}-(1+\beta)^{n}\left(1+\beta^{4 t+2}\right)^{n}\right]
\end{aligned}
$$

which, since $\alpha^{2}=\alpha+1, \beta^{2}=\beta+1$ and by Lemma 2.2 equals

$$
\begin{aligned}
& \frac{1}{\alpha-\beta}\left[\alpha^{2 n} 5^{\frac{n}{2}} \alpha^{(2 t+1) n} F_{2 t+1}^{n}-(-1)^{n} \beta^{2 n} 5^{\frac{n}{2}} \beta^{(2 t+1) n} F_{2 t+1}^{n}\right] \\
& =5^{\frac{n}{2}} F_{2 t+1}^{n}\left[\frac{\alpha^{(2 t+3) n}-(-1)^{n} \beta^{(2 t+3) n}}{\alpha-\beta}\right]
\end{aligned}
$$

Thus,

$$
\sum_{0 \leq i, j, k \leq n}\left(\begin{array}{l}
n \\
i
\end{array}\right)\left(\begin{array}{l}
i \\
j
\end{array}\right)\left(\begin{array}{l}
j \\
k
\end{array}\right) F_{i+(4 t+1) j+k}=F_{2 t+1}^{n}\left\{\begin{array}{cl}
5^{\frac{n}{2}} F_{(2 t+3) n} & \text { if } n \text { is even } \\
5^{\frac{n-1}{2}} L_{(2 t+3) n} & \text { if } n \text { is odd }
\end{array}\right.
$$

as claimed.

\section{Alternating analogues of binomial triple sums with the Fibonacci numbers}

Now we will give alternating analogues of the results given in the previous section. We shall present our results but for later use, we recall with two auxiliary lemmas from [5].

Lemma 3.1. Let t be any integer.

i) For odd $k$,

$$
\begin{aligned}
& (-1)^{t} \alpha^{-k(2 t+1)}-\alpha^{k}=(-1)^{t+1} V_{k(t+1)} \beta^{k t} \\
& (-1)^{t} \beta^{-k(2 t+1)}-\beta^{k}=(-1)^{t+1} V_{k(t+1)} \alpha^{k t}
\end{aligned}
$$

ii) For even k,

$$
\begin{aligned}
\alpha^{-k(2 t+1)}-\alpha^{k} & =-\sqrt{\Delta} U_{k(t+1)} \beta^{k t}, \\
\beta^{-k(2 t+1)}-\beta^{k} & =\sqrt{\Delta} U_{k(t+1)} \alpha^{k t} .
\end{aligned}
$$

Lemma 3.2. Let t be an integer.

i) For odd $k$,

$$
\begin{aligned}
& (-1)^{t} \alpha^{k(1-2 t)}-\alpha^{k}=(-1)^{t} U_{k t} \beta^{k(t-1)} \sqrt{\Delta} \\
& (-1)^{t} \beta^{k(1-2 t)}-\beta^{k}=(-1)^{t+1} U_{k t} \alpha^{k(t-1)} \sqrt{\Delta}
\end{aligned}
$$

ii) For even k,

$$
\begin{aligned}
& \alpha^{k(1-2 t)}-\alpha^{k}=-U_{k t} \beta^{k(t-1)} \sqrt{\Delta} \\
& \beta^{k(1-2 t))}-\beta^{k}=U_{k t} \alpha^{k(t-1)} \sqrt{\Delta}
\end{aligned}
$$


Theorem 3.3. For nonnegative integer $n$ and integer $t$

$$
\begin{aligned}
& \text { (i) } \sum_{0 \leq i, j, k \leq n}\left(\begin{array}{l}
n \\
i
\end{array}\right)\left(\begin{array}{l}
i \\
j
\end{array}\right)\left(\begin{array}{l}
j \\
k
\end{array}\right)(-1)^{i} F_{i+(4 t+2) j+k}=(-1)^{n} F_{2 t+3}^{n}\left\{\begin{array}{cc}
5^{\frac{n}{2}} F_{(2 t+2) n} & \text { if } n \text { is even } \\
5^{\frac{n-1}{2}} L_{(2 t+2) n} \text { if } n \text { is odd }
\end{array}\right. \\
& \text { (ii) } \sum_{0 \leq i, j, k \leq n}\left(\begin{array}{l}
n \\
i
\end{array}\right)\left(\begin{array}{l}
i \\
j
\end{array}\right)\left(\begin{array}{l}
j \\
k
\end{array}\right)(-1)^{i} F_{i+4 t j+k}=(-1)^{n} L_{2 t+2}^{n} F_{(2 t+1) n} \\
& \text { (iii) } \sum_{0 \leq i, j, k \leq n}\left(\begin{array}{l}
n \\
i
\end{array}\right)\left(\begin{array}{l}
i \\
j
\end{array}\right)\left(\begin{array}{l}
j \\
k
\end{array}\right)(-1)^{i} F_{i+j}=(-1)^{n}\left\{\begin{array}{cc}
5^{\frac{n}{2}} F_{2 n} \quad \text { if n is even } \\
5^{\frac{n-1}{2}} L_{2 n} \text { if } n \text { is odd }
\end{array}\right. \\
& \text { (iv) } \sum_{0 \leq i, j, k \leq n}\left(\begin{array}{l}
n \\
i
\end{array}\right)\left(\begin{array}{l}
i \\
j
\end{array}\right)\left(\begin{array}{l}
j \\
k
\end{array}\right)(-1)^{i} F_{i+j+4 k}=(-1)^{n} 5^{n} F_{3 n} \\
& \text { (v) } \sum_{0 \leq i, j, k \leq n}\left(\begin{array}{l}
n \\
i
\end{array}\right)\left(\begin{array}{l}
i \\
j
\end{array}\right)\left(\begin{array}{l}
j \\
k
\end{array}\right)(-1)^{i} F_{2 i+j+k}=(-1)^{n} 3^{n} F_{3 n} \\
& \text { (vi) } \sum_{0 \leq i, j, k \leq n}\left(\begin{array}{l}
n \\
i
\end{array}\right)\left(\begin{array}{l}
i \\
j
\end{array}\right)\left(\begin{array}{l}
j \\
k
\end{array}\right)(-1)^{i} F_{3 i+j+k}=(-1)^{n} 5^{n} F_{3 n} \\
& \text { (vii) } \sum_{0 \leq i, j, k \leq n}\left(\begin{array}{l}
n \\
i
\end{array}\right)\left(\begin{array}{l}
i \\
j
\end{array}\right)\left(\begin{array}{l}
j \\
k
\end{array}\right)(-1)^{i} F_{j+k}=(-1)^{n} F_{3 n}
\end{aligned}
$$

Proof. We only prove the first claim. The others could be similarly proven. Consider the left-hand side of the first claim, by the Binet formula, equals

$$
\frac{1}{\alpha-\beta} \sum_{0 \leq i, j, k \leq n}\left(\begin{array}{l}
n \\
i
\end{array}\right)\left(\begin{array}{l}
i \\
j
\end{array}\right)\left(\begin{array}{l}
j \\
k
\end{array}\right)(-1)^{i}\left[\alpha^{i+(4 t+2) j+k}-\beta^{i+(4 t+2) j+k}\right],
$$

which, by Lemma 2.1 and since $\alpha^{2}=\alpha+1$ and $\beta^{2}=\beta+1$, equals

$$
\begin{aligned}
& \frac{1}{\alpha-\beta}\left[\left(1-\alpha-\alpha^{4 t+3}-\alpha^{4 t+4}\right)^{n}-\left(1-\beta-\beta^{4 t+3}-\beta^{4 t+4}\right)^{n}\right] \\
& =\frac{1}{\alpha-\beta}\left[\left(1-\alpha-\alpha^{4 t+5}\right)^{n}-\left(1-\beta-\beta^{4 t+5}\right)^{n}\right] \\
& =\frac{1}{\alpha-\beta}\left[\left(-\alpha^{-1}-\alpha^{4 t+5}\right)^{n}-\left(-\beta^{-1}-\beta^{4 t+5}\right)^{n}\right] \\
& =\frac{(-1)^{n}}{\alpha-\beta}\left[\left(\alpha^{4 t+5}+\alpha^{-1}\right)^{n}-\left(\beta^{4 t+5}+\beta^{-1}\right)^{n}\right]
\end{aligned}
$$

which, from Lemma 3.2 by taking $k=-1$ and $t=2 t+3$, equals

$$
(-1)^{n} 5^{\frac{n}{2}} F_{2 t+3}^{n}\left[\frac{\alpha^{(2 t+2) n}-(-1)^{n} \beta^{(2 t+2) n}}{\alpha-\beta}\right] \text {. }
$$

Finally, we write

$$
\sum_{0 \leq i, j, k \leq n}\left(\begin{array}{l}
n \\
i
\end{array}\right)\left(\begin{array}{l}
i \\
j
\end{array}\right)\left(\begin{array}{l}
j \\
k
\end{array}\right)(-1)^{i} F_{i+(4 t+2) j+k}=(-1)^{n} F_{(2 t+3)}^{n}\left\{\begin{array}{cl}
5^{\frac{n}{2}} F_{(2 t+2) n} & \text { if } n \text { is even } \\
5^{\frac{n-1}{2}} L_{(2 t+2) n} & \text { if } n \text { is odd }
\end{array}\right.
$$

as claimed. 
Now, we give the other results.

Theorem 3.4. For nonnegative integer $n$ and integer $t$

(i) $\sum_{0 \leq i, j, k \leq n}\left(\begin{array}{l}n \\ i\end{array}\right)\left(\begin{array}{l}i \\ j\end{array}\right)\left(\begin{array}{l}j \\ k\end{array}\right)(-1)^{j} F_{i+(4 t+3) j+k}=(-1)^{n} F_{2 t+2}^{n}\left\{\begin{array}{cl}5^{\frac{n}{2}} F_{(2 t+4) n} & \text { if } n \text { is even } \\ 5^{\frac{n-1}{2}} L_{(2 t+4) n} & \text { if } n \text { is odd }\end{array}\right.$

(ii) $\sum_{0 \leq i, j, k \leq n}\left(\begin{array}{l}n \\ i\end{array}\right)\left(\begin{array}{l}i \\ j\end{array}\right)\left(\begin{array}{l}j \\ k\end{array}\right)(-1)^{j} F_{i+(4 t+1) j+k}=(-1)^{n} L_{2 t+1}^{n} F_{(2 t+3) n}$

(iii) $\sum_{0 \leq i, j, k \leq n}\left(\begin{array}{l}n \\ i\end{array}\right)\left(\begin{array}{l}i \\ j\end{array}\right)\left(\begin{array}{l}j \\ k\end{array}\right)(-1)^{j} F_{i+j+t k}=(-1)^{n} F_{(t+2) n}$

(iv) $\sum_{0 \leq i, j, k \leq n}\left(\begin{array}{l}n \\ i\end{array}\right)\left(\begin{array}{l}i \\ j\end{array}\right)\left(\begin{array}{l}j \\ k\end{array}\right)(-1)^{j} F_{i+2 j+k}=(-1)^{n} F_{11 n}$

(v) $\sum_{0 \leq i, j, k \leq n}\left(\begin{array}{l}n \\ i\end{array}\right)\left(\begin{array}{l}i \\ j\end{array}\right)\left(\begin{array}{l}j \\ k\end{array}\right)(-1)^{j} F_{3 i+j+k}=(-1)^{n} 3^{n} F_{3 n}$

(vi) $\sum_{0 \leq i, j, k \leq n}\left(\begin{array}{l}n \\ i\end{array}\right)\left(\begin{array}{l}i \\ j\end{array}\right)\left(\begin{array}{l}j \\ k\end{array}\right)(-1)^{j} F_{4 i+j+k}=(-1)^{n} 5^{n} F_{3 n}$

(vii) $\sum_{0 \leq i, j, k \leq n}\left(\begin{array}{l}n \\ i\end{array}\right)\left(\begin{array}{l}i \\ j\end{array}\right)\left(\begin{array}{l}j \\ k\end{array}\right)(-1)^{j} F_{i+k}=(-1)^{n} F_{n}$

Proof. We only prove the second claim. The others could be similarly proven. Consider the left-hand side of the second claim, by the Binet formula, equals

$$
\frac{1}{\alpha-\beta} \sum_{0 \leq i, j, k \leq n}\left(\begin{array}{l}
n \\
i
\end{array}\right)\left(\begin{array}{l}
i \\
j
\end{array}\right)\left(\begin{array}{l}
j \\
k
\end{array}\right)(-1)^{j}\left[\alpha^{i+(4 t+1) j+k}-\beta^{i+(4 t+1) j+k}\right],
$$

which, by Lemma 2.1 equals

$$
\begin{aligned}
& \frac{1}{\alpha-\beta}\left[\left(1+\alpha-\alpha^{4 t+2}-\alpha^{4 t+3}\right)^{n}-\left(1+\beta-\beta^{4 t+2}-\beta^{4 t+3}\right)^{n}\right] \\
& =\frac{1}{\alpha-\beta}\left[(1+\alpha)^{n}\left(1-\alpha^{4 t+2}\right)^{n}-(1+\beta)\left(1-\beta^{4 t+2}\right)^{n}\right]
\end{aligned}
$$

which, by Lemma 2.2 and since $\alpha^{2}=\alpha+1$ and $\beta^{2}=\beta+1$, equals

$$
\begin{aligned}
& \frac{1}{\alpha-\beta}\left[\alpha^{2 n}(-1)^{n} \alpha^{(2 t+1) n} L_{2 t+1}^{n}-\beta^{2 n}(-1)^{n} \beta^{(2 t+1) n} L_{2 t+1}^{n}\right] \\
= & \frac{(-1)^{n} L_{2 t+1}^{n}}{\alpha-\beta}\left[\alpha^{(2 t+3) n}-\beta^{(2 t+3) n}\right] \\
= & (-1)^{n} L_{2 t+1}^{n} F_{(2 t+3) n}
\end{aligned}
$$

as claimed. Hence, the proof of the second claim finishes.

By using previously given lemmas the next results could be proved similarly. 
Theorem 3.5. For nonnegative integer $n$ and integer $t$

(i) $\sum_{0 \leq i, j, k \leq n}\left(\begin{array}{l}n \\ i\end{array}\right)\left(\begin{array}{l}i \\ j\end{array}\right)\left(\begin{array}{l}j \\ k\end{array}\right)(-1)^{k} F_{i+(4 t+2) j+k}=(-1)^{n} F_{2 t}^{n}\left\{\begin{array}{cc}5^{\frac{n}{2}} F_{(2 t+2) n} & \text { if } n \text { is even } \\ 5^{\frac{n-1}{2}} L_{(2 t+2) n} & \text { if } n \text { is odd }\end{array}\right.$

(ii) $\sum_{0 \leq i, j, k \leq n}\left(\begin{array}{l}n \\ i\end{array}\right)\left(\begin{array}{l}i \\ j\end{array}\right)\left(\begin{array}{l}j \\ k\end{array}\right)(-1)^{k} F_{i+4 t j+k}=(-1)^{n} L_{(2 t-1)}^{n} F_{(2 t+1) n}$

(iii) $\sum_{0 \leq i, j, k \leq n}\left(\begin{array}{l}n \\ i\end{array}\right)\left(\begin{array}{l}i \\ j\end{array}\right)\left(\begin{array}{l}j \\ k\end{array}\right)(-1)^{k} F_{i+j+3 k}=(-1)^{n}\left\{\begin{array}{cl}5^{\frac{n}{2}} F_{2 n} & \text { if } n \text { is even } \\ 5^{\frac{n-1}{2}} L_{2 n} & \text { if } n \text { is odd }\end{array}\right.$

(iv) $\sum_{0 \leq i, j, k \leq n}\left(\begin{array}{l}n \\ i\end{array}\right)\left(\begin{array}{l}i \\ j\end{array}\right)\left(\begin{array}{l}j \\ k\end{array}\right)(-1)^{k} F_{i+5 j+k}=(-1)^{n} 2^{n} F_{3 n}$

(v) $\sum_{0 \leq i, j, k \leq n}\left(\begin{array}{l}n \\ i\end{array}\right)\left(\begin{array}{l}i \\ j\end{array}\right)\left(\begin{array}{l}j \\ k\end{array}\right)(-1)^{k} F_{i+j+4 k}=(-1)^{n} 3^{n} F_{3 n}$

(vi) $\sum_{0 \leq i, j, k \leq n}\left(\begin{array}{l}n \\ i\end{array}\right)\left(\begin{array}{l}i \\ j\end{array}\right)\left(\begin{array}{l}j \\ k\end{array}\right)(-1)^{k} F_{i+j+2 k}=(-1)^{n} F_{n}$

(vii) $\sum_{0 \leq i, j, k \leq n}\left(\begin{array}{l}n \\ i\end{array}\right)\left(\begin{array}{l}i \\ j\end{array}\right)\left(\begin{array}{l}j \\ k\end{array}\right)(-1)^{k} F_{i+3 j+k}=(-1)^{n} F_{n}$

(viii) $\sum_{0 \leq i, j, k \leq n}\left(\begin{array}{l}n \\ i\end{array}\right)\left(\begin{array}{l}i \\ j\end{array}\right)\left(\begin{array}{l}j \\ k\end{array}\right)(-1)^{k} F_{i+j}=F_{2 n}$

(ix) $\sum_{0 \leq i, j, k \leq n}\left(\begin{array}{l}n \\ i\end{array}\right)\left(\begin{array}{l}i \\ j\end{array}\right)\left(\begin{array}{l}j \\ k\end{array}\right)(-1)^{k} F_{t i+j+k}=0$

Theorem 3.6. For nonnegative integer $n$ and integer $t$

(i) $\sum_{0 \leq i, j, k \leq n}\left(\begin{array}{l}n \\ i\end{array}\right)\left(\begin{array}{l}i \\ j\end{array}\right)\left(\begin{array}{l}j \\ k\end{array}\right)(-1)^{i+j} F_{i+4 t j+k}=F_{2 t+2}^{n}\left\{\begin{array}{cc}5^{\frac{n}{2}} F_{(2 t+1) n} & \text { if } n \text { is even } \\ 5^{\frac{n-1}{2}} L_{(2 t+1) n} & \text { if } n \text { is odd }\end{array}\right.$

(ii) $\sum_{0 \leq i, j, k \leq n}\left(\begin{array}{l}n \\ i\end{array}\right)\left(\begin{array}{l}i \\ j\end{array}\right)\left(\begin{array}{l}j \\ k\end{array}\right)(-1)^{i+j} F_{i+(4 t+2) j+k}=F_{(2 t+2) n} L_{2 t+3}^{n}$

(iii) $\sum_{0 \leq i, j, k \leq n}\left(\begin{array}{l}n \\ i\end{array}\right)\left(\begin{array}{l}i \\ j\end{array}\right)\left(\begin{array}{l}j \\ k\end{array}\right)(-1)^{i+j} F_{2 i+j+k}=\left\{\begin{array}{cc}5^{\frac{n}{2}} F_{3 n} & \text { if } n \text { is even } \\ 5^{\frac{n-1}{2}} L_{3 n} & \text { if } n \text { is odd }\end{array}\right.$

(iv) $\sum_{0 \leq i, j, k \leq n}\left(\begin{array}{l}n \\ i\end{array}\right)\left(\begin{array}{l}i \\ j\end{array}\right)\left(\begin{array}{l}j \\ k\end{array}\right)(-1)^{i+j} F_{i+j+3 k}=5^{n} F_{2 n}$

(v) $\sum_{0 \leq i, j, k \leq n}\left(\begin{array}{l}n \\ i\end{array}\right)\left(\begin{array}{l}i \\ j\end{array}\right)\left(\begin{array}{l}j \\ k\end{array}\right)(-1)^{i+j} F_{j+k}=F_{3 n}$ 
Theorem 3.7. For nonnegative integer $n$ and integer $t$

(i) $\sum_{0 \leq i, j, k \leq n}\left(\begin{array}{l}n \\ i\end{array}\right)\left(\begin{array}{l}i \\ j\end{array}\right)\left(\begin{array}{l}j \\ k\end{array}\right)(-1)^{j+k} F_{i+4 t j+k}=F_{2 t-1}^{n}\left\{\begin{array}{cc}5^{\frac{n}{2}} F_{(2 t+1) n} & \text { if } n \text { is even } \\ 5^{\frac{n-1}{2}} L_{(2 t+1) n} & \text { if } n \text { is odd }\end{array}\right.$

(ii) $\sum_{0 \leq i, j, k \leq n}\left(\begin{array}{l}n \\ i\end{array}\right)\left(\begin{array}{l}i \\ j\end{array}\right)\left(\begin{array}{l}j \\ k\end{array}\right)(-1)^{j+k} F_{i+(4 t+2) j+k}=F_{(2 t+2) n} L_{2 t}^{n}$

(iii) $\sum_{0 \leq i, j, k \leq n}\left(\begin{array}{l}n \\ i\end{array}\right)\left(\begin{array}{l}i \\ j\end{array}\right)\left(\begin{array}{l}j \\ k\end{array}\right)(-1)^{j+k} F_{3 i+j+k}=\left\{\begin{array}{cc}5^{\frac{n}{2}} F_{3 n} & \text { if } n \text { is even } \\ 5^{\frac{n-1}{2}} L_{3 n} & \text { if } n \text { is odd }\end{array}\right.$

(iv) $\sum_{0 \leq i, j, k \leq n}\left(\begin{array}{l}n \\ i\end{array}\right)\left(\begin{array}{l}i \\ j\end{array}\right)\left(\begin{array}{l}j \\ k\end{array}\right)(-1)^{j+k} F_{i+3 j+k}=F_{4 n}$

(v) $\sum_{0 \leq i, j, k \leq n}\left(\begin{array}{l}n \\ i\end{array}\right)\left(\begin{array}{l}i \\ j\end{array}\right)\left(\begin{array}{l}j \\ k\end{array}\right)(-1)^{j+k} F_{i+5 j+k}=2^{n} F_{4 n}$

(vi) $\sum_{0 \leq i, j, k \leq n}\left(\begin{array}{l}n \\ i\end{array}\right)\left(\begin{array}{l}i \\ j\end{array}\right)\left(\begin{array}{l}j \\ k\end{array}\right)(-1)^{j+k} F_{i+j+t k}=F_{(t+2) n}$

Theorem 3.8. For nonnegative integer $n$ and integer $t$

(i) $\sum_{0 \leq i, j, k \leq n}\left(\begin{array}{l}n \\ i\end{array}\right)\left(\begin{array}{l}i \\ j\end{array}\right)\left(\begin{array}{l}j \\ k\end{array}\right)(-1)^{i+k} F_{i+(4 t+3) j+k}=F_{2 t+2}^{n}\left\{\begin{array}{cc}5^{\frac{n}{2}} F_{(2 t+1) n} & \text { if } n \text { is even } \\ 5^{\frac{n-1}{2}} L_{(2 t+1) n} & \text { if } n \text { is odd }\end{array}\right.$

(ii) $\sum_{0 \leq i, j, k \leq n}\left(\begin{array}{l}n \\ i\end{array}\right)\left(\begin{array}{l}i \\ j\end{array}\right)\left(\begin{array}{l}j \\ k\end{array}\right)(-1)^{i+k} F_{i+(4 t+1) j+k}=F_{2 t n} L_{2 t+1}^{n}$

(iii) $\sum_{0 \leq i, j, k \leq n}\left(\begin{array}{l}n \\ i\end{array}\right)\left(\begin{array}{l}i \\ j\end{array}\right)\left(\begin{array}{l}j \\ k\end{array}\right)(-1)^{i+k} F_{i+j+2 k}=\left\{\begin{array}{cc}5^{\frac{n}{2}} F_{n} & \text { if } n \text { is even } \\ 5^{\frac{n-1}{2}} L_{n} & \text { if } n \text { is odd }\end{array}\right.$

(iv) $\sum_{0 \leq i, j, k \leq n}\left(\begin{array}{l}n \\ i\end{array}\right)\left(\begin{array}{l}i \\ j\end{array}\right)\left(\begin{array}{l}j \\ k\end{array}\right)(-1)^{i+k} F_{i+j+3 k}=3^{n} F_{2 n}$

(v) $\sum_{0 \leq i, j, k \leq n}\left(\begin{array}{l}n \\ i\end{array}\right)\left(\begin{array}{l}i \\ j\end{array}\right)\left(\begin{array}{l}j \\ k\end{array}\right)(-1)^{i+k} F_{i+j}=-F_{n}$

(vi) $\sum_{0 \leq i, j, k \leq n}\left(\begin{array}{l}n \\ i\end{array}\right)\left(\begin{array}{l}i \\ j\end{array}\right)\left(\begin{array}{l}j \\ k\end{array}\right)(-1)^{i+k} F_{i+k}=-F_{2 n}$

Theorem 3.9. For nonnegative integer $n$ and integer $t$

(i) $\sum_{0 \leq i, j, k \leq n}\left(\begin{array}{l}n \\ i\end{array}\right)\left(\begin{array}{l}i \\ j\end{array}\right)\left(\begin{array}{l}j \\ k\end{array}\right)(-1)^{i+j+k} F_{i+(4 t+1) j+k}=(-1)^{n} F_{2 t+1}^{n}\left\{\begin{array}{cc}5^{\frac{n}{2}} F_{2 t n} & \text { if } n \text { is even } \\ 5^{\frac{n-1}{2}} L_{2 t n} & \text { if } n \text { is odd }\end{array}\right.$ 


$$
\begin{aligned}
& \text { (ii) } \sum_{0 \leq i, j, k \leq n}\left(\begin{array}{l}
n \\
i
\end{array}\right)\left(\begin{array}{l}
i \\
j
\end{array}\right)\left(\begin{array}{l}
j \\
k
\end{array}\right)(-1)^{i+j+k} F_{i+(4 t+3) j+k}=(-1)^{n} F_{(2 t+1) n} L_{2 t+2}^{n} \\
& \text { (iii) } \sum_{0 \leq i, j, k \leq n}\left(\begin{array}{l}
n \\
i
\end{array}\right)\left(\begin{array}{l}
i \\
j
\end{array}\right)\left(\begin{array}{l}
j \\
k
\end{array}\right)(-1)^{i+j+k} F_{i+2 j+k}=(-1)^{n} 2^{n} F_{n} \\
& \text { (iv) } \sum_{0 \leq i, j, k \leq n}\left(\begin{array}{l}
n \\
i
\end{array}\right)\left(\begin{array}{l}
i \\
j
\end{array}\right)\left(\begin{array}{l}
j \\
k
\end{array}\right)(-1)^{i+j+k} F_{i+k}=(-1)^{n} F_{n} \\
& \text { (v) } \sum_{0 \leq i, j, k \leq n}\left(\begin{array}{l}
n \\
i
\end{array}\right)\left(\begin{array}{l}
i \\
j
\end{array}\right)\left(\begin{array}{l}
j \\
k
\end{array}\right)(-1)^{i+j+k} F_{5 i+j+k}=(-1)^{n} 5^{n} F_{3 n} \\
& \text { (vi) } \sum_{0 \leq i, j, k \leq n}\left(\begin{array}{l}
n \\
i
\end{array}\right)\left(\begin{array}{l}
i \\
j
\end{array}\right)\left(\begin{array}{l}
j \\
k
\end{array}\right)(-1)^{i+j+k} F_{2 i+j+k}=(-1)^{n} F_{3 n} \\
& \text { (vii) } \sum_{0 \leq i, j, k \leq n}\left(\begin{array}{l}
n \\
i
\end{array}\right)\left(\begin{array}{l}
i \\
j
\end{array}\right)\left(\begin{array}{l}
j \\
k
\end{array}\right)(-1)^{i+j+k} F_{4 i+j+k}=(-1)^{n} 3^{n} F_{3 n} \\
& \text { (viii) } \sum_{0 \leq i, j, k \leq n}\left(\begin{array}{l}
n \\
i
\end{array}\right)\left(\begin{array}{l}
i \\
j
\end{array}\right)\left(\begin{array}{l}
j \\
k
\end{array}\right)(-1)^{i+j+k} F_{i+j+2 k}=(-1)^{n} 3^{n} F_{n} \\
& \text { (ix) } \sum_{0 \leq i, j, k \leq n}\left(\begin{array}{l}
n \\
i
\end{array}\right)\left(\begin{array}{l}
i \\
j
\end{array}\right)\left(\begin{array}{l}
j \\
k
\end{array}\right)(-1)^{i+j+k} F_{i+j}=-F_{n}
\end{aligned}
$$

Finally, we would like to mention that we consider the sums including Fibonacci numbers rather than Lucas numbers. Similarly sums including the Lucas numbers could be computed and it could be shown that they have nice multiplication forms.

\section{References}

[1] Carlitz, L. (1978). Some classes of Fibonacci sums. The Fibonacci Quarterly, 16, 411-426.

[2] Chen, L., \& Wang, X. (2019). The power sums involving Fibonacci polynomials and their applications. Symmetry, 11(5), 635.

[3] Deveci, O., \& Artun, G. (2019). On the Adjacency-Jacobsthal numbers. Communications in Algebra, 47(11), 4520-4532.

[4] Deveci, O., \& Karaduman, E. (2017). On the Padovan p-numbers. Hacettepe Journal of Mathematics and Statistics, 46(4), 579-592.

[5] Kılıç, E. (2016). Some classes of alternating weighted binomial sums. Analele Ştiințifice ale Universitătii “Al. I. Cuza” Iaşi. Mat. (N.S.), 3, 835-843.

[6] Kılıç, E., Akkuş, İ., Ömür, N., \& Ulutaş, Y. T. (2015). Formulas for binomial sums including powers of Fibonacci and Lucas numbers. UPB Scientific Bulletin, Series A, 77, 69-78. 
[7] Kılıç, E., \& Arıkan, T. (2018). Double binomial sums and double sums related with certain linear recurrences of various order. Chiang Mai Journal of Science, 45, 1569-1577.

[8] Kılıç, E., \& Belbachir, H. (2017). Generalized double binomial sums families by generating functions. Utilitas Mathematica, 104, 161-174.

[9] Kılıç, E., Ömür, N., \& Ulutaş, Y. T. (2011). Binomial sums whose coefficients are products of terms of binary sequences. Utilitas Mathematica, 84, 45-52.

[10] Kılıç, E., \& Taşdemir, F. (2019). On binomial double sums with Fibonacci and Lucas numbers-I. Ars Combinatoria, 144, 173-185.

[11] Kılıç, E., \& Taşdemir, F. (2019). On binomial double sums with Fibonacci and Lucas numbers-II. Ars Combinatoria, 144, 345-354.

[12] Koparal, S., \& Ömür, N. (2016). On congruences related to central binomial coefficients, harmonic and Lucas numbers. Turkish Journal of Mathematics, 40, 973-985.

[13] Layman, J. W. (1977). Certain general binomial-Fibonacci sums. The Fibonacci Quarterly, $15,362-366$.

[14] Ömür, N., \& Duran, Ö. (2020). On binomial triple sums involving Fibonacci and Lucas numbers. Honam Mathematical Journal, 42, 49-62.

[15] Ömür, N., \& Şener, C. D. (2017). On identities for sequences of binomial sums with the terms of sequences $\left\{u_{k n}\right\}$ and $\left\{v_{k n}\right\}$. European Journal of Pure and Applied Mathematics, $10,506-515$.

[16] Piejko, K. (2018). On $k$-distance Pell numbers and $((k-1) A,(k-1) B, k C)$-edge coloured graphs. Ars Combinatoria, 139, 197-215.

[17] Seibert, J., \& Trojovsky, P. (2005). On some identities for the Fibonomial coefficients. Mathematica Slovaca, 55, 9-19.

[18] Shannon, A. G. (2007). Some generalized rising binomial coefficients. Notes on Number Theory and Discrete Mathematics, 13(1), 25-30.

[19] Shannon, A. G., \& Deveci, O. (2020). Rising binomial coefficients-type 1: extensions of Carlitz and Riordan. Advanced Studies on Contemporary Mathematics, 30(2), 263-268.

[20] Taşdemir, F. (2020). Binomial triple sums with the Lucas numbers. Punjab University Journal of Mathematics, 52, 37-43.

[21] Taşdemir, F., \& Toska, T. G. (2020). Formulas for binomial double sums related to Lucas numbers. Ars Combinatoria, 152, 235-246.

[22] Vajda, S. (1989). Fibonacci \& Lucas Numbers, and the Golden Section, John Wiley \& Sons, Inc., New York. 